\title{
Gadofullerene nanoparticles extend survival rate and down-regulate thrombin expression in orthotopic pancreatic cancer
}

\author{
Xue $\mathrm{Li}^{1,2}$, Mingming Zhen ${ }^{1,2^{*}}$, Meilan $\mathrm{Yu}^{3}$, Chen Zhou ${ }^{1,2}$, Lei $\mathrm{Li}^{1,2}$, Chunying Shu ${ }^{1,2}$, Chunru Wang ${ }^{1,2^{*}}$ and \\ Chunli Bai ${ }^{1,2}$
}

\begin{abstract}
Pancreatic cancer is a devastating malignant disease with 5-year survival rate less than $8 \%$. The impenetrable desmoplastic stroma of pancreatic tissue and serious side-effects of existing drugs hinder the effective treatment for pancreatic carcinoma. Thus, it is imperative to exploit much more safe and efficient methods to prolong the survival of pancreatic cancer patients. In this study, we explored a superior anti-pancreatic cancer strategy based on gadofullerene nanoparticles (GFNPs) using an orthotopic human pancreatic carcinoma (PANC-1) tumor model. It was demonstrated that GFNPs could efficiently suppress orthotopic pancreatic cancer in a dose manner, and significantly extend the survival rate of tumor-bearing mice. Of note, the proteomic profiling of tumor tissues revealed that GFNPs ameliorated the coagulation cascade dysfunction and downregulated the thrombin expression in pancreatic tumor tissues. The regulation of abnormal thrombin by GFNPs was validated in vitro and in vivo. More importantly, GFNPs suppressed orthotopic pancreatic cancer with negligible adverse effects, superior to the widely recognized clinical antipancreatic cancer drug, gemcitabine. Together, this study provides a promising therapeutic for intractable pancreatic cancer as well as a potential to alleviate the cancer-associated thromboembolic diseases.
\end{abstract}

Keywords: gadofullerene nanoparticles, pancreatic cancer, survival rate, coagulation cascade, proteomics

\section{INTRODUCTION}

Pancreatic cancer is a devastating malignant disease and a leading cause of cancer morbidity and mortality throughout the world $[1,2]$. The 5 -year survival rate of the patients with pancreatic cancer is less than $8 \%$, one of the lowest among all cancers [3]. As is known to all, it is hard to cure pancreatic cancer. One reason is that pancreatic tissues consist of impenetrable desmoplastic stroma, inducing the limitation of drug penetration. In addition, the serious side-effects and drug resistance of existing drugs (e.g., gemcitabine) further hinder the effective treatment for pancreatic carcinoma [4,5]. Although some emerging therapeutic strategies have been developed for cancer treatment such as chemotherapy, targeting therapy, gene therapy, and immunotherapy, the outcomes remain unsatisfactory [6-8]. Therefore, it is still urgent to explore innovative and effective therapeutic approaches for pancreatic cancer.

It is acknowledged that pancreatic cancer remains a hypercoagulability state with a close association with the blood coagulation cascade [9-12]. And it has been reported that thrombin plays a crucial role in the pathogenesis, progression, and metastasis of pancreatic carcinoma. Due to hypercoagulability and diffuse intravascular coagulation, the thromboembolic diseases including deep venous thrombosis, pulmonary embolism, portal vein thrombosis, and arterial thromboembolism are important causes of death except for pancreatic cancer itself $[13,14]$. Cancer-associated thromboembolic disease has become one of the uppermost complications of pancreatic cancer, which is always accompanied by grave clinical consequences. Therefore, the cancer-associated thromboembolic disease should also be taken into consideration along with anti-pancreatic cancer therapeutics.

Recently, advances in nanotechnology have been leveraged for cancer therapeutics, particularly involving improving drug delivery and regulating tumor microenvironment [15-18]. Fullerene and gadofullerene, as versatile carbon nanomaterials, have been widely exploited in biomedical fields $[19,20]$. They were diversely used for chemotherapeutic injury protection, magnetic resonance imaging and type II diabetes treatment [2128]. In particular, functionalized $\mathrm{Gd} @ \mathrm{C}_{82}$ exhibited various potentials in tumor treatment and metastasis inhibition by disrupting tumor vasculatures and activating immune system [2932].

Based on the outstanding features of gadofullerene, herein, we explored it to treat pancreatic cancer using amino acid-functionalized gadofullerene nanoparticles (GFNPs). Excitedly, we found that GFNPs could efficiently suppress orthotopic pancreatic cancer with low adverse effects and prominently extended survival. Importantly, we elucidated the intrinsic proteome changes altered by GFNPs through proteomic profiles. Of note, it revealed that GFNPs could down-regulate thrombin and ameliorated blood coagulation dysfunction, presenting the

\footnotetext{
${ }^{1}$ Beijing National Laboratory for Molecular Sciences, Key Laboratory of Molecular Nanostructure and Nanotechnology, CAS Research/Education Center for Excellence in Molecular Sciences, Institute of Chemistry, Chinese Academy of Sciences, Beijing 100190, China

${ }^{2}$ University of Chinese Academy of Sciences, Beijing 100049, China

${ }^{3}$ College of life Science, Zhejiang Sci-Tech University, Hangzhou 310018, China

*Corresponding authors (emails: zhenmm@iccas.ac.cn (Zhen M); crwang@iccas.ac.cn (Wang C))
} 
potential to ameliorate pancreatic cancer-associated thromboembolic disease.

\section{EXPERIMENTAL SECTION}

\section{Preparation and characterization of GFNPs}

Water-soluble amino acid-functionalized GFNPs were prepared as the previously reported reference [33]. Briefly, the suspension comprised of $50 \mathrm{mg}$ of $\mathrm{Gd} @ \mathrm{C}_{82}, 3.6 \mathrm{~g}$ of $\beta$-alanine and $50 \mathrm{~mL}$ of $\mathrm{NaOH}(14 \%)$ was vigorously stirred at $80^{\circ} \mathrm{C}$ until the black particles gradually dissolved and turned into a brown solution. And the solution was purified by precipitation with ethanol, centrifugation, and dialysis. The GFNPs were further characterized by Fourier transform infrared spectroscopy (FT-IR, Nicolet iN10TM, Thermo Scientific, USA), and atomic force microscope (AFM, NanoWizerd 4 Nanoscience, JPK, Germany). The size and Zeta-potential of the GFNPs in water were characterized by dynamic light scattering (DLS, Malvern, UK) at $25^{\circ} \mathrm{C}$.

\section{Animal experiments}

The animal experiments were performed with $\mathrm{BALB} / \mathrm{c}$ nude mice. Female $\mathrm{BALB} / \mathrm{c}$ nude mice with body weights ranging from 18 to $20 \mathrm{~g}$ were purchased from Perking University Laboratory Animal Center, China. The mice were housed in a temperature-controlled, ventilated, and standardized animal room. And the animal experiments were conducted by a professional technician. All mice were cared for according to the standards of the Guide for the Care and Use of Laboratory Animal, the Experimental Animal Center of Institute of Chemistry, Chinese Academy of Sciences (CAS). And all the experimental protocols involving live animals were reviewed and approved by the Animal Ethics Committee of Institute of Chemistry, CAS.

PANC-1 cells were cultured to establish an orthotopic model of pancreatic cancer. After washing with phosphate-buffered saline (PBS), the PANC-1 cells were subcutaneously injected into the armpits of mice. The subcutaneous tumors were used as the source of tissue for orthotopic implantation onto the pancreas.

Forty BALB/c nude mice after inoculation were divided into five groups as follows: (1) Saline, $10 \mathrm{~mL} \mathrm{~kg}^{-1}$ per day; (2) Gemcitabine (GEM), $133.5 \mu \mathrm{mol} \mathrm{kg}^{-1}$ per two days; (3) GFNPs-L, $4 \mu \mathrm{mol} \mathrm{kg}^{-1}$ per day; (4) GFNPs-M, $8 \mu \mathrm{mol} \mathrm{kg}^{-1}$ per day; (5) GFNPs-H, $16 \mu \mathrm{mol} \mathrm{kg}{ }^{-1}$ per day. The drug administration was initiated two days after tumor implantation via intraperitoneal injection, and lasted for three weeks according to the dosages as mentioned above. GEM was taken as a positive drug injected every other day for three weeks. Exploratory laparotomy was conducted to observe tumor growth twice during the experiment. At last, mice were sacrificed after anesthesia by avertin. The blood was obtained, and tumor tissues were collected, photographed, and preserved as soon as possible. Some tissues were fixed in $4 \%$ formalin at once, and some tissues were immediately iced in liquid nitrogen and stored at $-80^{\circ} \mathrm{C}$ for further investigation.

\section{Proteomic profiles}

The proteomic profiling was conducted according to the procedure as follows: protein extraction, trypsin digestion, labeling, liquid chromatography-mass spectrometry (LC-MS)/MS, pro- tein identification, and bioinformatical analysis.

After the treatment by GFNPs $\left(16 \mu \mathrm{mol} \mathrm{kg}{ }^{-1} \mathrm{~d}^{-1}\right)$ for three weeks, tumor tissues in control $(n=3)$ and GFNPs $(n=3)$ groups were fully ground into powder in liquid nitrogen. And the lysis buffer $\left(8 \mathrm{~mol} \mathrm{~L}^{-1}\right.$ urea, $50 \mathrm{mmol} \mathrm{L}^{-1}$ Tris, $\mathrm{pH} 8.0,1 \%$ protease inhibitor cocktail) was added to the powder and sonicated three times on ice using a high-intensity ultrasonic processor (Scientz, China). Finally, the protein extraction solutions were collected from the supernatant after centrifugation at $20,000 \times \mathrm{g}$ at $4^{\circ} \mathrm{C}$ for $10 \mathrm{~min}$. The protein concentration was determined using the bicinchoninic acid (BCA) Protein Assay Kit (Thermal Fisher Scientific) according to the manufacturer's instructions.

For trypsin digestion, the protein solutions with certain protein contents were reduced by $5 \mathrm{mmol} \mathrm{L}^{-1}$ dithiothreitol for $60 \mathrm{~min}$ at $37^{\circ} \mathrm{C}$. And then the reduced protein solution was incubated with $15 \mathrm{mmol} \mathrm{L}^{-1}$ iodoacetamide at room temperature in darkness for $30 \mathrm{~min}$. At last, the protein was digested by trypsin overnight with a 1:50 trypsin-to-protein mass ratio after being precipitated and cleaned with acetone twice.

After trypsin digestion, the peptide fragments were desalted using a Sep-Pak C18 column (Waters, USA), vacuum-dried, and re-dissolved in $500 \mathrm{mmol} \mathrm{L}^{-1}$ triethylammonium bicarbonate. The labeling reagent (iTRAQI8plex, AB Sciex, USA) was thawed and dissolved with isopropanol. The peptide fragments were mixed and incubated with the labeling reagent at room temperature for $1 \mathrm{~h}$, followed by freeze-drying.

The labeled peptides were dissolved with $0.1 \%$ trifluoroacetic acid and $0.5 \%$ acetonitrile, and then the labeled peptide solution was injected to the desalination column (Sep-Pak C18 column, Waters, USA). After being eluted by trifluoroacetic acid and acetonitrile with different concentrations, the desalted labeled peptides were collected and freeze-dried.

Next, the high-pH reverse-phase high-performance liquid

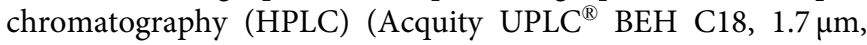
$2.1 \mathrm{~mm} \times 50 \mathrm{~mm}$, Waters, USA) was used to separate the tryptic peptide fractions. The peptide powder was dissolved with $10 \mathrm{mmol} \mathrm{L}^{-1}$ ammonium formate $(\mathrm{pH} 10.0)$ and then separated by the column as mentioned above with a flow rate of $250 \mu \mathrm{L} \mathrm{min}^{-1}$ and wavelength of $215 \mathrm{~nm}$. The elution was comprised of solution A (10 $\mathrm{mmol} \mathrm{L}^{-1}$ ammonium formate, $\left.\mathrm{pH} 10.0\right)$ and solution $\mathrm{B}\left(10 \mathrm{mmol} \mathrm{L}^{-1}\right.$ ammonium formate, $90 \%$ acetonitrile, $\mathrm{pH} 10.0$ ). The gradient was started with $45 \%$ solution $\mathrm{B}$ for $35 \mathrm{~min}$, and then increased from $45 \%$ to $80 \%$ solution B in $37 \mathrm{~min}$ and held at $80 \%$ solution B for $40 \mathrm{~min}$, decreased from $80 \%$ to $5 \%$ solution B in $42 \mathrm{~min}$ and held at $5 \%$ solution B for $45 \mathrm{~min}$.

The peptides were analyzed by Nano (Acclaim PepMap C18, $250 \mathrm{~mm} \times 75 \mathrm{~mm}$, Thermo Scientific, USA), followed by tandem MS/MS in the positive ion mode. The elution comprised of solution A ( $0.1 \%$ formic acid) and solution B ( $0.1 \%$ formic acid in $98 \%$ acetonitrile) with a flow rate of $300 \mathrm{~nL} \mathrm{~min}^{-1}$. The gradient comprised of $5 \%$ solution B for $5 \mathrm{~min}, 30 \%$ solution B for $45 \mathrm{~min}, 80 \%$ solution B for $50 \mathrm{~min}$, and $5 \%$ solution B for $55 \mathrm{~min}$. For the MS analysis, the electrospray voltage applied was $1.8 \mathrm{kV}$, and the ion fragmentation mode was high-energy collision dissociation (HCD).

The resulting MS data were analyzed for protein identification using the Mascot Database System (Version 2.3.2, Matrix Science). The fixed modification mainly included carbamidomethyl on cysteine and iTRAQ 8plex (N-term), and the variable mod- 
ification was mainly about oxidation on Met. The trypsin was the cleavage enzyme that allows up to 2 missing cleavages. The algorithm was Percolator with false discovery rate (FDR) $\leq 1 \%$.

At last, the bioinformatical analysis was conducted by Gene Ontology (GO) and Kyoto Encyclopedia of Genes and Genomes (KEGG) database equipped with the platform of UniprotKB (https://www.uniprot.org/uniprot/), STING v.10 online database platform (http://string-db.org/), WebGestalt (http://bioinfo. vanderbilt.edu/webgestalt/) and KEGG mapper (https://www. genome.jp/kegg/tool/map_pathway2.html).

\section{Thrombin activity assay}

Thrombin activity assay was carried out according to the previous reference [34]. Briefly, different concentrations of GFNPs (final concentration: $0.025,0.05,0.1 \mathrm{mmol} \mathrm{L}^{-1}$ ) or argatroban (final concentration: $2 \mu \mathrm{mol} \mathrm{L}{ }^{-1}$, positive control) were added into Tris- $\mathrm{HCl}$ buffer $\left(50 \mathrm{mmol} \mathrm{L}^{-1}, \mathrm{pH} 7.4,7.5 \mathrm{mmol} \mathrm{L}^{-1}\right.$ ethylene diamine tetraacetic acid). Forty microliter of those solutions were added into 96-well plates and incubated for $2 \mathrm{~min}$. And then, thrombin solution $\left(30 \mu \mathrm{L}, 50 \mathrm{U} \mathrm{mL}^{-1}\right)$ was introduced into the solution above, followed by culturing for $2 \mathrm{~min}$ at $37^{\circ} \mathrm{C}$. Next, the chromogenic substitute S-2238 $\left(30 \mu \mathrm{L}, 1.5 \mathrm{mmol} \mathrm{L}^{-1}\right)$ was added into the solution above after being preheated at $37^{\circ} \mathrm{C}$.
After the addition, the plate was immediately monitored using a microplate reader (TECAN, Switzerland) at $405 \mathrm{~nm}$ at the time points of $1,2,3,5,8,10,12,15,20,25 \mathrm{~min}$. The experiments were repeated more than three times.

\section{Data analysis}

The data were expressed as means \pm standard deviations. The statistical analysis was conducted using Student's $t$-test or oneway analysis of variance (ANOVA) analysis by software of IBM SPSS Statistics 25. For the results, ${ }^{*}$ means $P<0.05$ and ${ }^{* *}$ means $P<0.01$ compared with the saline or control group.

\section{RESULTS}

\section{The synthesis and characterization of GFNPs}

The amino acid derivatives of GFNPs were obtained via a solidliquid reaction in alkali aqueous solution as our reported method (Fig. 1a) [33]. Here, $\beta$-alanine was used to modify gadofullerenes due to its water-solubility, short chain length and simple structure. After purification by dialysis, GFNPs and the reactants of solid $\mathrm{Gd} @ \mathrm{C}_{82}$ and $\beta$-alanine were characterized by FT-IR. As shown in Fig. 1b, the characteristic absorption peaks were marked. GFNPs possessed the major peaks of carbon cages
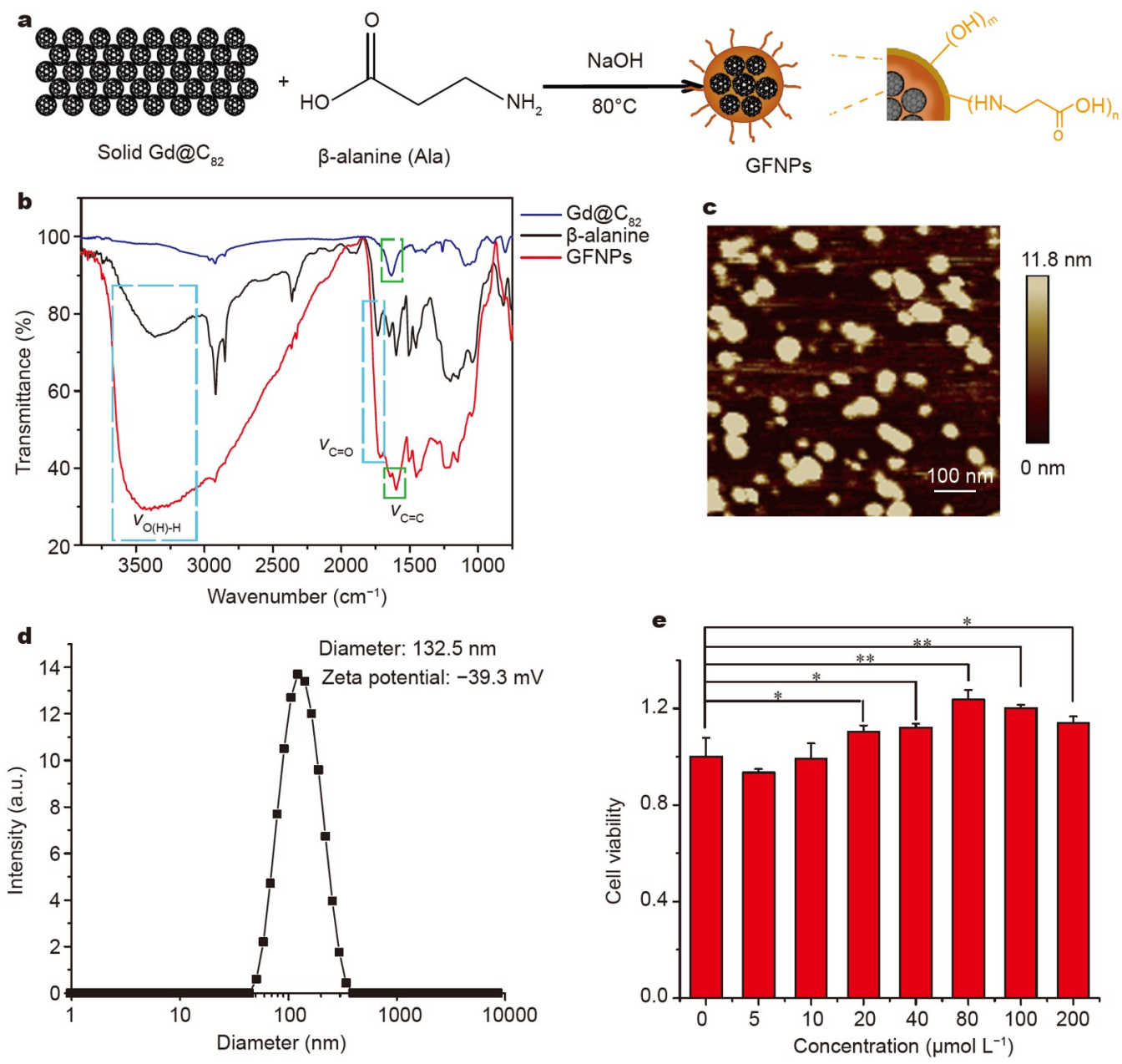

Figure 1 The synthesis and characterization of GFNPs. (a) Schematic diagram for synthesis of GFNPs. (b) FT-IR spectra of solid Gd@C 82 , $\beta$-alanine and GFNPs. (c) AFM of GFNPs. (d) The hydrodynamic diameter and zeta-potential of GFNPs measured by DLS. (e) Cell viabilities of PANC-1 cells incubated with GFNPs at different concentrations. ${ }^{*}$ means $P<0.05$ and ${ }^{* *}$ means $P<0.01$. 
of solid $\mathrm{Gd} @ \mathrm{C}_{82}$, such as the skeletal vibration bands of $-\mathrm{C}=\mathrm{C}$ at $1600 \mathrm{~cm}^{-1}$. The mean peaks of 3428 and $1712 \mathrm{~cm}^{-1}$ were ascribed to the characteristic vibration bands of $-\mathrm{O}(\mathrm{N}) \mathrm{H}$ and $-\mathrm{C}=\mathrm{O}$, suggesting the presence of alanine. Then, the AFM was conducted to observe the morphology of GFNPs, revealing that the average diameter was $50.9 \pm 3.2 \mathrm{~nm}$ and the height was $10.6 \pm$ $1.1 \mathrm{~nm}$ (Fig. 1c). Besides, the size distribution and Zeta potential of GFNPs in water were measured by DLS. The hydrodynamic diameter of GFNPs was about $132.5 \mathrm{~nm}$, and the Zeta potential was approximately $-39.3 \mathrm{mV}$ (Fig. 1d). In addition, the cytotoxicity of GFNPs in vitro was evaluated. As shown in Fig. 1e, there was no significant reduction of cell viabilities, suggesting negligible cytotoxicity of GFNPs in vitro.

\section{Anti-tumor effects of GFNPs on orthotopic pancreatic cancer} As aforementioned in our previous work, the GFNPs were highly accumulated into pancreatic tissue through intraperitoneal (i.p.) injection [35]. Inspired by their versatile properties, we explored GFNPs to treat orthotopic pancreatic tumors. Two days after PANC-1 implantation, GFNPs were injected intraperitoneally daily for three weeks (Fig. 2a). The commonly used anti-pancreatic cancer drug GEM was administrated every other day as a positive drug. After three weeks of treatment, the mice were sacrificed and tumors were collected. As shown in Fig. 2b, c, GFNPs could significantly reduce the volume and weight of tumor compared with the saline control group in a dose-dependent manner. In particular, the tumor inhibitory rate of GFNPs with the dosage of $16 \mu \mathrm{mol} \mathrm{kg}{ }^{-1}$ was about $67.85 \%$, comparable to that of GEM (Fig. 2d). Consistently, the hematoxylin and eosin (H\&E) staining of tumor tissues after GFNPs treatment revealed observable cellular disruption without intact nuclei and cytoplasm, suggesting the anti-tumor efficacy of GFNPs was extremely impressive (Fig. 2e). It is worth noting that our results also depicted the significant increase of animal survival by GFNPs treatment. The median survival time for tumor-bearing mice treated with saline was 34-38 days, which was extended to 49 days after GFNPs treatment (Fig. 2f). These data indicated that GFNPs could remarkably inhibit the orthotopic pancreatic tumor growth with extended survival time.

\section{The proteomics profiling of orthotopic pancreatic tumor after GFNPs treatment}

To expound the intrinsic molecular alterations of the anti-tumor effects, a Tandem Mass Tag (TMT)-labeled quantitative proteomics was conducted to identify and quantify the protein expression changes in orthotopic pancreatic tumor after treatment by GFNPs. Tumor tissues were lysed and proteins were extracted, denatured, and digested. After labelling, the peptides were identified by LC-MS and analyzed by bioinformatical methods (Fig. 3a). In total, about 4894 proteins were identified and about 4817 proteins were quantified (Table S1). Compared with the saline-treated group, there were significant changes in

a

\begin{tabular}{|c|c|c|}
\hline 2 days & & reatment for three weeks \\
\hline & & Saline, i.p., $10 \mathrm{~mL} \mathrm{~kg}^{-1} \mathrm{~d}^{-1}$ \\
\hline $\begin{array}{l}\text { Tumor } \\
\text { implantation }\end{array}$ & Treatments & $\begin{array}{l}\text { GEM, i.p., } 133.5 \mu \mathrm{mol} \mathrm{kg}^{-1} \text { per two days } \\
\text { GFNPs-L/M/H, i.p., } 4 / 8 / 16 \mu \mathrm{mol} \mathrm{kg}^{-1} \mathrm{~d}^{-1}\end{array}$ \\
\hline
\end{tabular}

b

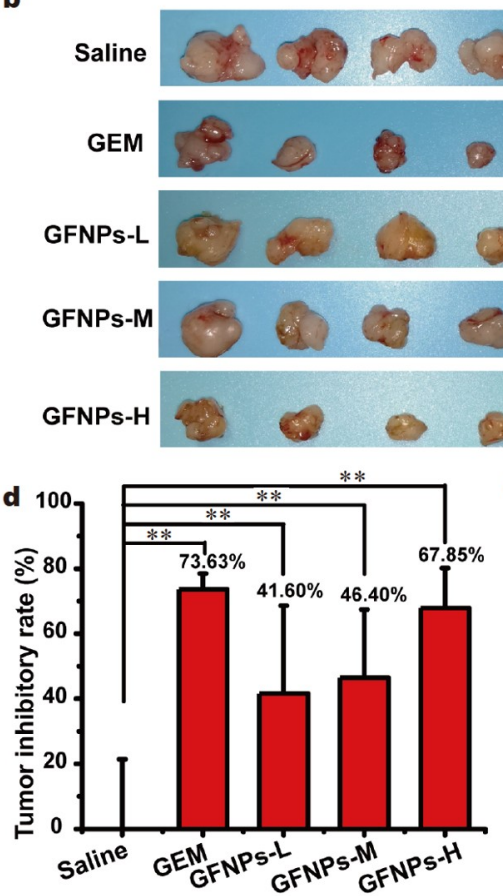

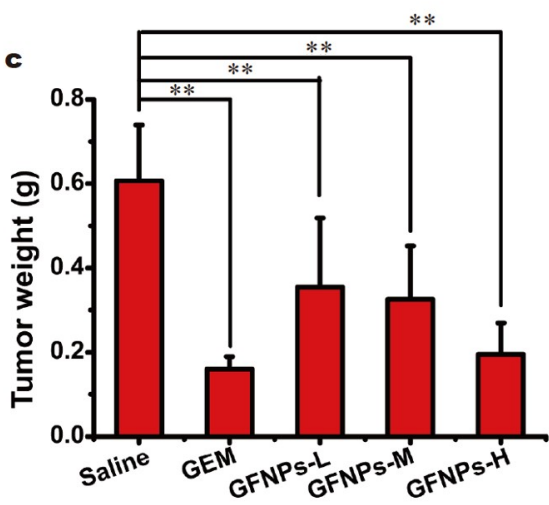
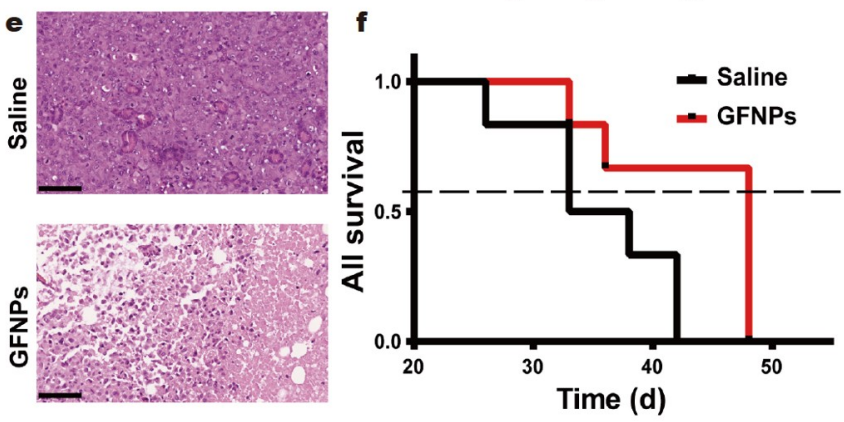

Figure 2 Anti-tumor effects of GFNPs on orthotopic pancreatic tumor. (a) Schematic diagram of anti-tumor treatment process of GFNPs. (b) Photographs of tumor tissues after treatment for three weeks in different groups. (c) Tumor weights after treatment for three weeks. (d) The tumor inhibitory rate in different groups. (e) The H\&E staining of tumor tissues in the groups of the saline and GFNPs. Scale bars are $100 \mu \mathrm{m}$. (f) All survival of tumor-bearing mice in saline and GFNPs-treated groups. ${ }^{* *}$ means $P<0.01$ compared with the saline group analyzed by ANOVA with Tukey test. 
a Extraction/Denature/Digestion

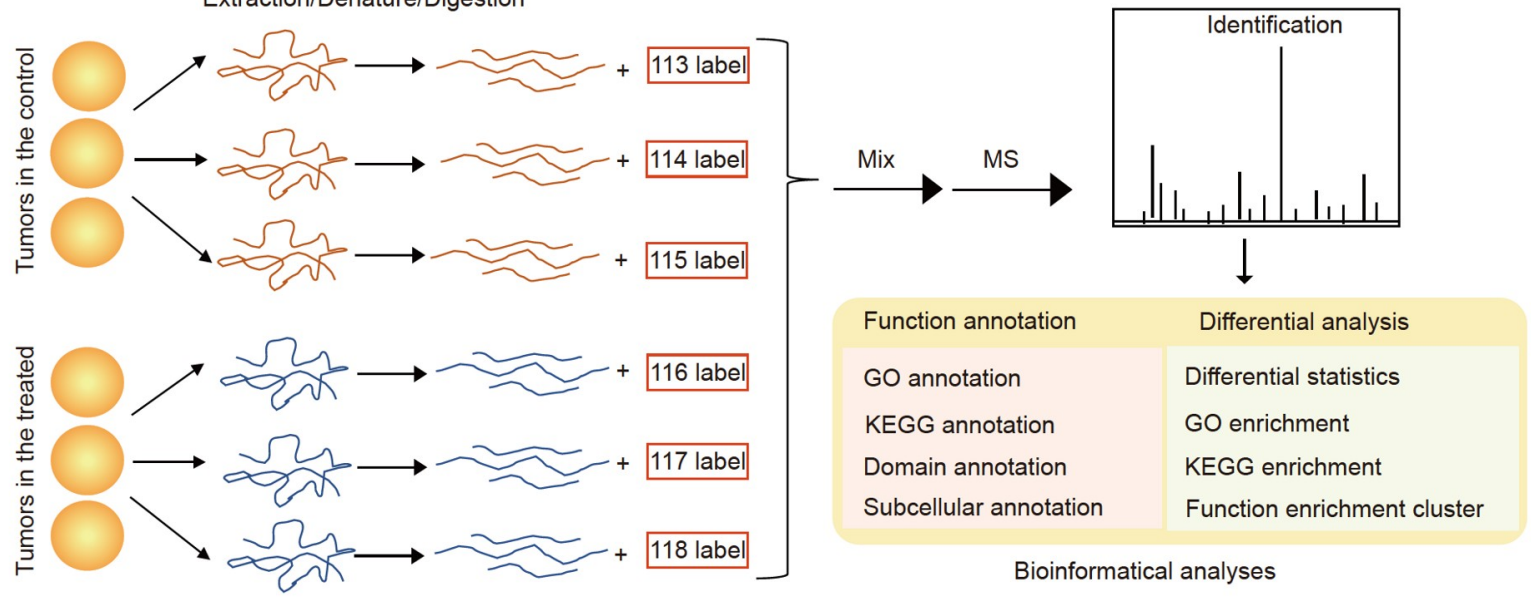

b

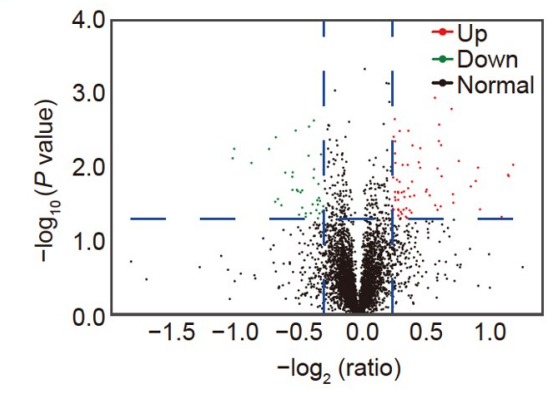

c

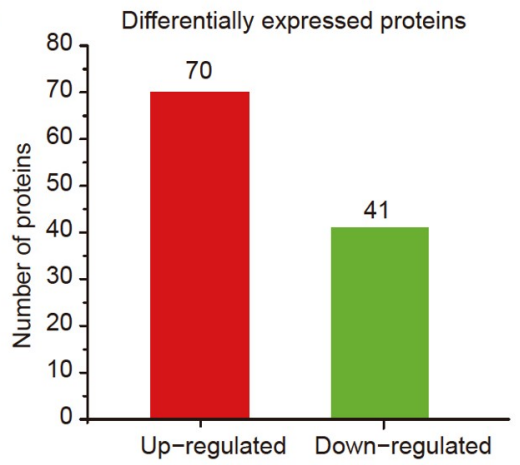

d Negative regulation of coagulation Negative regulation of blood coagulation Negative regulation of hemostasis Defense response Regulation of fibrinolysis Fibrinolysis Ganglioside metabolic process Ganglioside catabolic process -

Acute inflammatory response Negative regulation of wound healing

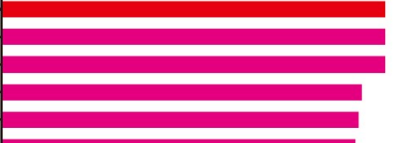

$-$

(20)

Extracellular space

Extracellular region

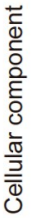
Extracellular region part Extracellular vesicle Extracellular organelle Extracellular exosome Vesicle Nucleosome Nucleosome
DNA packaging complex
Lysosome Nucleosome
DNA packaging complex
Lysosome (29) $+2$ Beta-N-acetylhexosaminidase activity
Enzyme inhibitor activity

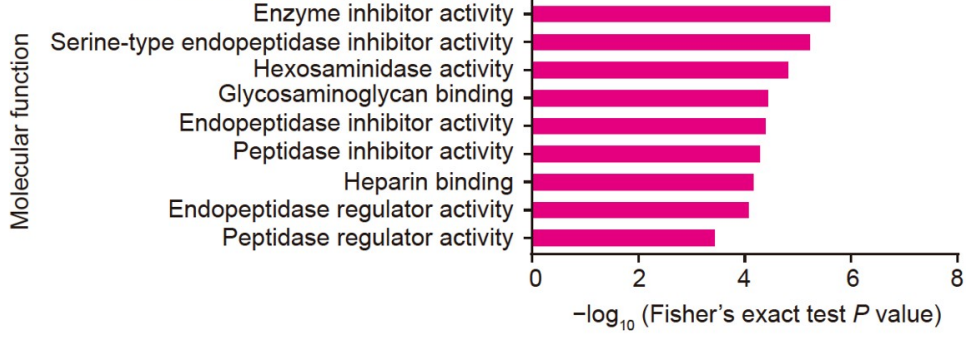

Figure 3 Tumor proteomic profiling. (a) The experimental workflow for the proteomic profiling of the pancreatic tumor tissues. (b) Volcano plot of proteins: red points refer to up-regulated DEPs, green points refer to down-regulated DEPs, and black points refer to non-DEPs. (c) The numbers of up- and down-regulated DEPs by GFNPs treatment. (d) GO analysis of the top 10 DEPs through the following three categories: biological process, cellular component and molecular function.

the identified proteins in tumors treated by GFNPs, as shown in the volcano plot in Fig. 3b. The proteomics depicted that there were 70 up-regulated and 41 down-regulated differential expression proteins (DEPs) with a fold change $>1.2$ and $P$-value $<0.05$ (Fig. 3c). Subsequently, those 114 differential proteins were annotated by GO annotation and clustered with GO term semantic similarity using the REVIGO server through the following three categories: biological process, cellular component and molecular function. GO analysis demonstrated that the most significantly differential proteins after treatment by GFNPs were primarily involved in the biological process of negative regula- tion of coagulation, and they mainly occurred on the extracellular space, with changing at the function of enzyme activity and enzyme inhibitor activity (Fig. 3d). Then, the networks of GO categories were analyzed by WebGestalt and Directed Acyclic Graphs (DAG) were presented (FDR < 0.05). Particular enrichment was found for coagulation-associated biological process (Figs S1 and S2), extracellular component (Fig. S3), enzyme and binding molecular function (Fig. S4). Additionally, the subcellular location distribution suggested that the differential proteins were mainly distributed in extracellular location (Fig. S5). 
According to the GO annotation, DEPs were classified in a heatmap as shown in Fig. 4. It was revealed that GFNPs regulated the protein expression mainly associated with coagulation, fibrinolysis, extracellular matrix organization, and activation of matrix metalloproteinases (MMP). The DEPs classified to carbohydrate metabolic, lipid metabolic process as well as the immune system and inflammatory response were also influenced by GFNPs treatment. To further uncover the functional regulation on biological systems of these differential proteins, the KEGG database was used to analyze the pathway enrichment. The differentially expressed protein enrichment of the KEGG pathway primarily focused on lysosome, systemic lupus erythematosus, alcoholism, complement and coagulation cascade, neuroactive ligand-receptor interaction, and so on (Fig. S6). Considering GO annotation and KEGG pathway enrichment as well as the tumor pathology, the lysosome, complement and coagulation cascade, Janus-activated kinase/signal transducers and activators of transcription (JAK-STAT) signaling pathway and neuroactive ligand-receptor interaction were primarily analyzed. The lysosome-associated proteins such as cathepsin (ctzb), hexa/b, legumain $(\operatorname{lgmn})$, and lysosomal acid lipase A (lipa), were expressed higher in GFNPs-treated tumors than the control (Fig. S7). In the coagulation cascade, the thrombin (F2), plasminogen (Plg), kallikrein (klkb1) and a2 antiplasmase (a2AP/Serpinf2) were down-regulated, and the alpha-1-antitrypsin (AIAT/Serpina1d) was up-regulated by GFNPs (Fig. S8). In the JAK-STAT signaling pathway, the expression of protein tyrosine phosphatase (TCPTP/ptpn2) was increased and Leukemia inhibitory factor receptor (Lifr) was decreased after GFNPs treatment (Fig. S9). In the pathway of neuroactive ligand-receptor interaction, the expression of proteinase-activated receptor substrate (PARRS) was decreased (Fig. S10). The typical proteins were further verified by western blot (WB) analysis as follows.

GFNPs down-regulated thrombin to alleviate coagulation cascade dysfunction of orthotopic pancreatic cancer

Combining the above analyses, we evaluated the protein expression in special pathways. The proteins such as cathepsin, thrombin, Plg, TCPTP, JAK, and ${ }^{\mathrm{pTyr}^{\mathrm{P}} 034 / 1035} \mathrm{JAK}$ were analyzed by WB. As shown in Fig. S11, the protein levels of cathepsin, Plg, TCPTP, and pJak1/Jak1 were almost unchanged between GFNPs-treated and untreated tumors. However, a central pleiotropic enzyme in coagulation system, thrombin, was obviously down-regulated by GFNPs treatment (Fig. 5a). Subsequently, the quantitative real-time polymerase chain reaction (Q-PCR) analysis was conducted and the results indicated that GFNPs observably decreased the mRNA expression of thrombin (Fig. 5b). We further evaluated the direct interaction between thrombin and GFNPs in vitro. The special thrombin inhibitor, argatroban, was used as a positive control. As shown in Fig. 5c, GFNPs significantly inhibited the thrombin activities, reducing the chromogenic reaction rate of thrombin substrate with a dose-dependent manner (Fig. 5d). All these results indicated that GFNPs could prominently suppress the expression of thrombin during orthotopic pancreatic cancer treatment.

\section{GFNPs exhibited low toxicity in vivo}

As a biomedical material for tumor therapeutics, the toxicity is a considerable issue. Here, the toxicity of GFNPs was evaluated by mouse weight, routine blood test, and histopathological examination. During the tumor treatment, we monitored the mouse weight, and no significant weight decreases were observed after treatment by GFNPs (Fig. 6a). However, mice treated by GEM

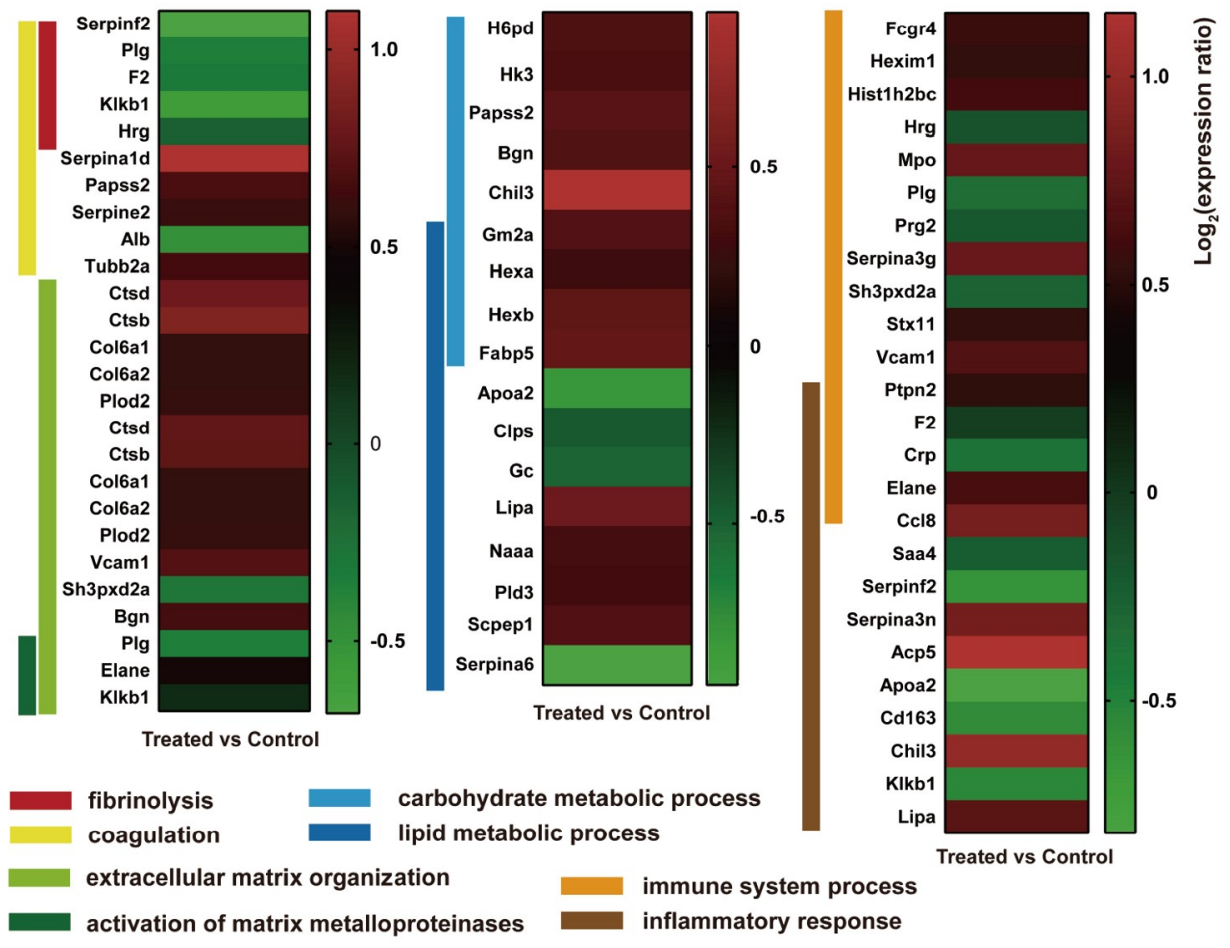

Figure 4 Heat map presentation of DEPs in the group of treated $v s$. control (with $P<0.05$, and with the 1.2 folds for up-regulation and 0.83 folds for downregulation). 

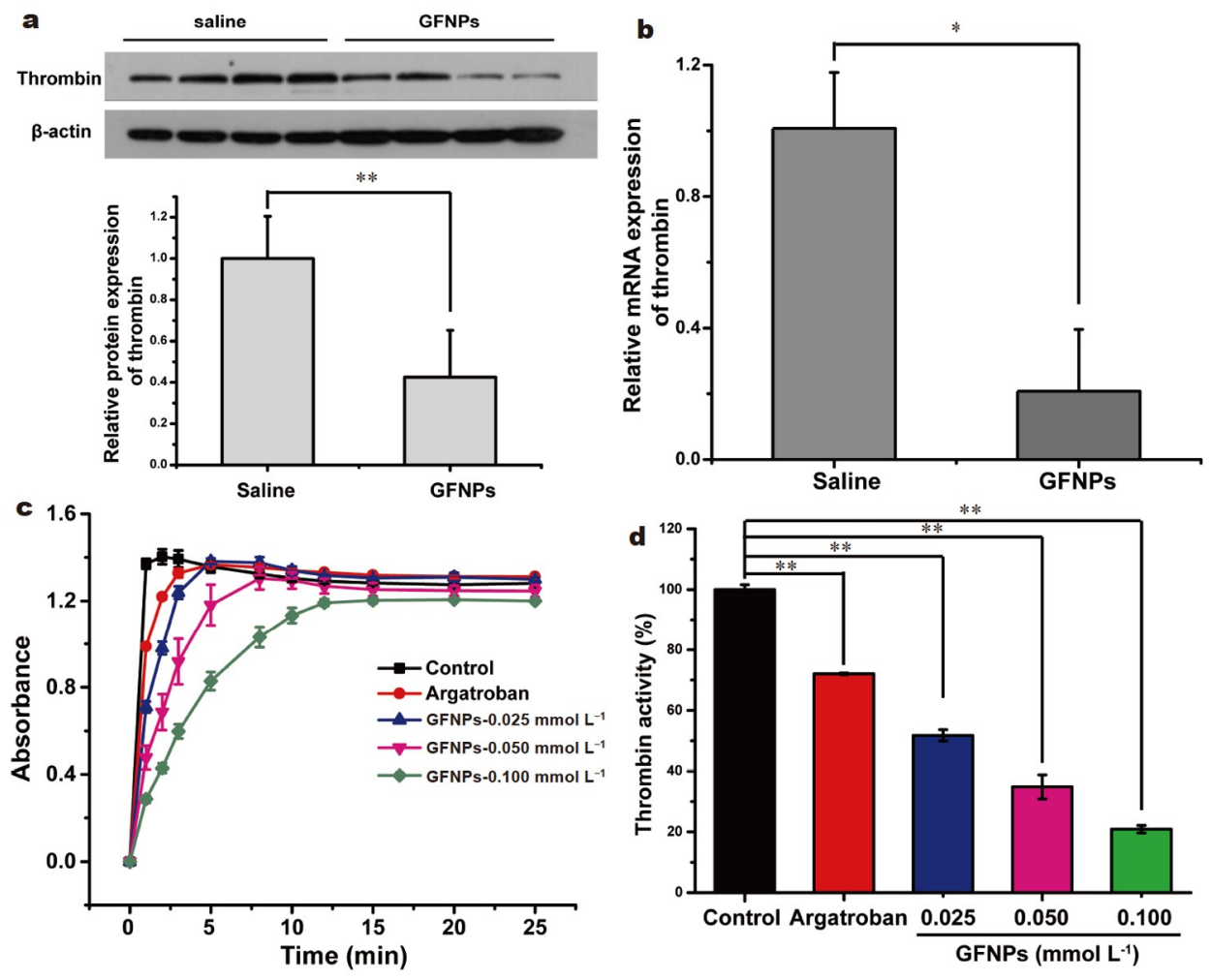

Figure 5 The effects of GFNPs on thrombin. (a) The protein expression of thrombin by WB. (b) The mRNA expression of thrombin by Q-PCR. (c) The absorbance of thrombin assay incubated with positive drug argatroban or GFNPs at different concentrations. (d) The thrombin activities calculated according to the first 1 min. ${ }^{*}$ means $P<0.05,{ }^{* *}$ means $P<0.01$ in $(\mathrm{a}, \mathrm{b})$ analyzed by Student's $t$-test. ${ }^{* *}$ means $P<0.01$ in $(\mathrm{d})$ analyzed by ANOVA with Tukey test.

lost weight seriously, suggesting the severe toxicity of GEM. Additionally, the content of white blood cells (WBC) exhibited normal levels in mice treated by GFNPs, instead of a remarkable decrease in mice treated by GEM (Fig. 6b). And the platelet (PLT) was reduced in GFNPs-treated mice, instead of a significant increase in mice treated by GEM. After treatment, the main organs of heart, liver, spleen, lung, kidney, and pancreas were stained with $\mathrm{H} \& \mathrm{E}$, and there were no obvious inflammation, cellular necrosis, and apoptosis in the group of GFNPs (Fig. 6c). However, in mice treated by GEM, some pathological changes were observed such as hepatic inflammatory infiltration, spleen lesions, and alveolar walls thickening. Although GEM could efficiently inhibit tumor growth, there are severe side effects after treatment. Inspiringly, GFNPs has no obvious toxicity in vivo.

\section{DISCUSSION}

Pancreatic cancer is still the deadliest carcinoma without standard therapeutic options in recent years [36]. To date, GEM remains the best chemotherapeutic medication available for treating pancreatic cancer with considerable efficacy. However, the treatment is always accompanied by dismal survival, adverse effects, and drug resistance [37,38]. Therefore, the therapeutic development is still urgent.

After functionalization, GFNPs exhibited excellent watersoluble and biocompatible properties. Previously, we found that GFNPs could be highly accumulated in the pancreas after intraperitoneal injection [35], and they also exhibited various applications in tumor treatment and anti-tumor metastasis by disrupting tumor vasculatures and activating the immune sys- tem. Encouragingly, in this work, we demonstrated that GFNPs could significantly inhibit tumor growth of orthotopic pancreatic cancer, and the inhibitory rate of GFNPs with the highest concentration was almost like that of GEM. In addition, it was demonstrated that most of the chemotherapeutic medications were remarkably toxic with the 5-year survival rate of the patients less than $8 \%$ [3]. Intriguingly, there was no obvious toxicity in tumor-bearing mice treated by GFNPs, including the less weight loss, hardly any damages to main organs and no reduction of WBCs. The survival rate, a major factor to evaluate the tumor therapeutic effect, was extended up to about 1.36 times after GFNPs treatment. Therefore, it was suggested that GFNPs may be a promising pancreatic cancer therapeutic medication with high anti-tumor effects, low side-effects, and extended survival.

To further elucidate the underlying changes of the anti-tumor effect of GFNPs, a global view of proteome changes was gained by GO annotation, subcellular localization, and KEGG analysis. After treatment by GFNPs, the significantly altered proteins mainly focused on the pathway of complement and the coagulation system. Obviously, the GO annotation in biological process category demonstrated that the DEPs mainly enriched in the process of negative regulation of blood coagulation. The WB analysis validated that the thrombin, a central enzyme of coagulation system, was significantly down-regulated by GFNPs treatment. Accordingly, the molecular function analysis indicated that proteins changed by GFNPs associated with enzyme inhibitor activity, enzyme activity, heparin binding, and so on, which was also related to coagulation system and consistent with the thrombin changes. The cellular component and subcellular 


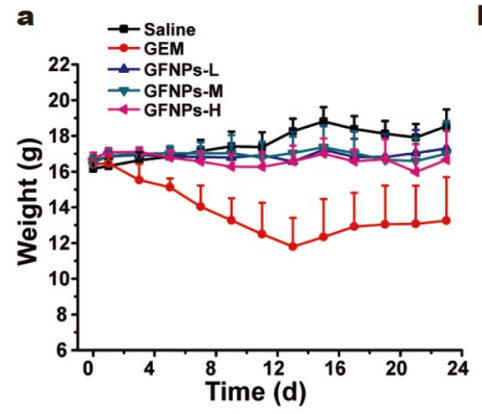

b

\begin{tabular}{clcc}
\hline Groups & $\begin{array}{c}\text { WBC } \\
\left(10^{9} \mathrm{~L}^{-1}\right)\end{array}$ & $\begin{array}{c}\text { RBC } \\
\left(10^{12} \mathrm{~L}^{-1}\right)\end{array}$ & $\begin{array}{c}\text { PLT } \\
\left(10^{9} \mathrm{~L}^{-1}\right)\end{array}$ \\
\hline Saline & $7.53 \pm 0.91$ & $8.58 \pm 1.28$ & $699.83 \pm 98.87$ \\
GEM & $4.85 \pm 1.19^{*}$ & $10.58 \pm 0.80$ & $1136.17 \pm 234.44^{* *}$ \\
GFNPs-L & $7.69 \pm 1.81^{\&}$ & $9.10 \pm 1.10$ & $743.00 \pm 112.05^{\& \&}$ \\
GFNPs-M & $8.40 \pm 1.05^{\&}$ & $8.91 \pm 0.28$ & $774.50 \pm 143.37^{8 \&}$ \\
GFNPs-H & $7.63 \pm 1.33^{\&}$ & $9.35 \pm 0.59$ & $785.50 \pm 62.24^{8 \&}$ \\
\hline
\end{tabular}
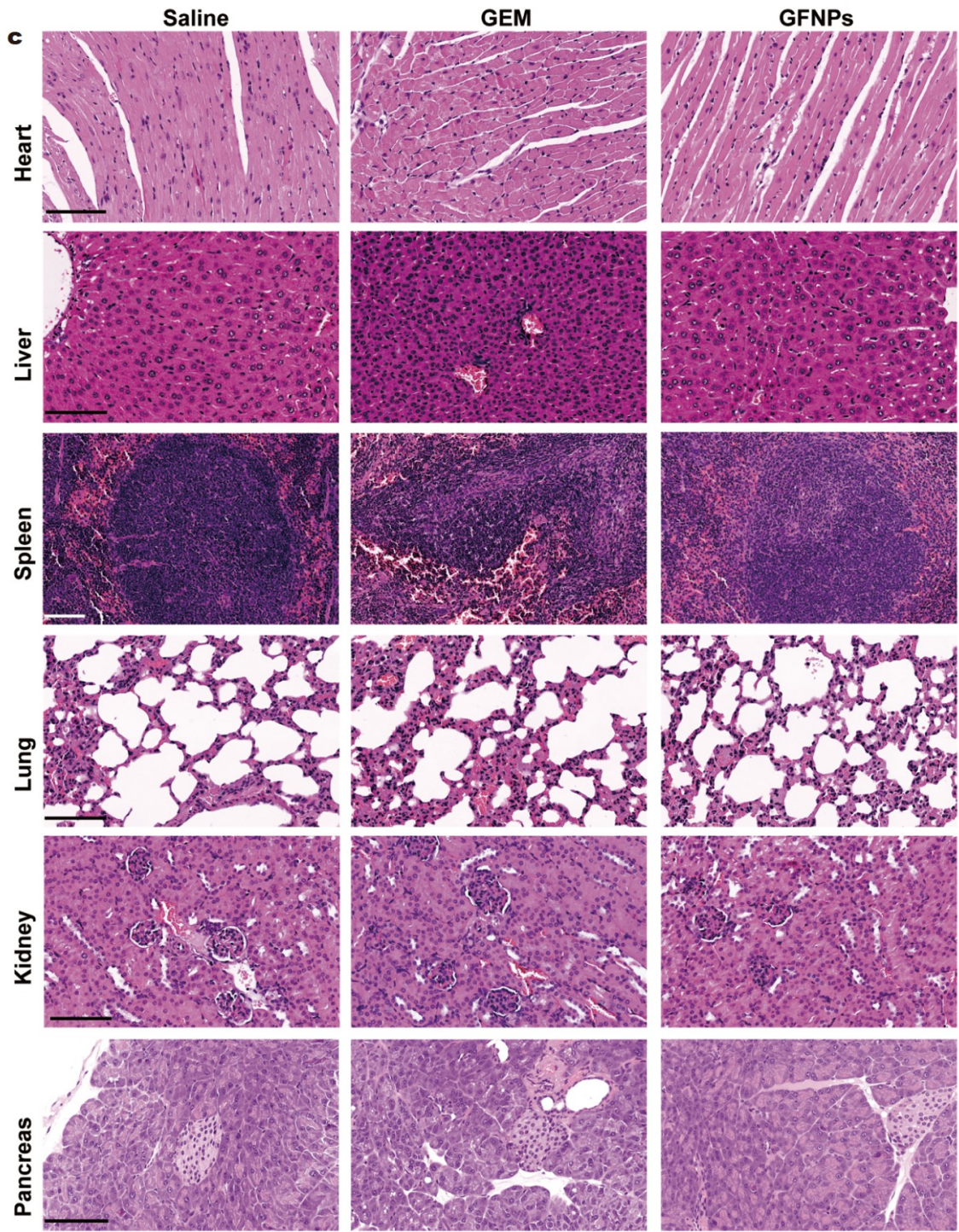

Figure 6 In vivo toxicity assessment of GFNPs treatment. (a) Body weights of mice with different treatments. (b) Routine blood test for WBC, red blood cells (RBC) and PLT of mice in different groups. (c) H\&E staining of heart, liver, spleen, lung, kidney and pancreas in groups treated by saline, GEM and GFNPs. Scale bars are $100 \mu \mathrm{m} .{ }^{*}$ means $P<0.05$ and ${ }^{* *}$ means $P<0.01$ compared with saline control group; ${ }^{\&}$ means $P<0.05$ and ${ }^{\& \&}$ means $P<0.01$ compared with GEM group analyzed by ANOVA with Tukey or Games-Howell tests.

localization analysis both confirmed that DEPs were localized in extracellular space and extracellular region, where the coagulation system acts [39]. The results indicated that GFNPs could modulate the coagulation system and down-regulate the thrombin expression in pancreatic cancer.

Evidence supports that pancreatic cancer retains a hypercoagulable state, resulting in an increased risk of thromboembolic disease along with substantial morbidity and mortality [40]. As the central factor in coagulation cascade, the thrombin was also abnormally up-regulated in pancreatic cancer [41]. Herein, we found that GFNPs could not only present excellent anti-tumor effects with neglectable toxicity and lengthened survival, but also inhibit thrombin expression, negatively regulate the coagulation system and thus improve the pancreatic cancer-associated 
thromboembolic disease. Studies showed that the activation of coagulation is not only considered as a simple epiphenomenon but might also be associated with promoting growth and angiogenesis of tumors, especially pancreatic cancer [13]. Thrombin could induce tumor angiogenesis, promoting tumor growth, and stimulating tumor implantation and metastasis [4244]. Emerging data implicated that anti-thrombin therapy has been used for cancer treatment associated with hypercoagulability, such as indirect thrombin inhibitor of heparin and direct thrombin inhibitor of dabigatran [45-47]. Therefore, in this work, the proteomics analysis oversaw the significant regulation of the coagulation cascade and inhibition of thrombin by GFNPs, which might be the great therapeutic effects on pancreatic cancer-associated thromboembolic disease as well as a cure mechanism of the effective tumor growth suppression.

\section{CONCLUSIONS}

In summary, we have devised a promising pancreatic cancer therapeutic based on functionalized GFNPs and uncovered the intrinsic proteome changes of tumor treated by GFNPs. After intraperitoneal injection, GFNPs significantly inhibited the orthotopic PANC-1 tumor growth, especially extending all survival of tumor-bearing mice. The proteomics profiling indicated that GFNPs mainly altered the signals associated with coagulation cascade. Of note, GFNPs inhibited thrombin activities and expression both in vivo and in vitro. Importantly, almost no visible toxicity was found after GFNPs treatment. Therefore, GFNPs could be a potential pancreatic cancer therapeutic with the prospect to ameliorate pancreatic cancer-associated thromboembolic disease.

\section{Received 16 April 2021; accepted 27 July 2021; published online 10 September 2021}

1 Ryan DP, Hong TS, Bardeesy N. Pancreatic adenocarcinoma. N Engl J Med, 2014, 371: 1039-1049

2 Vincent A, Herman J, Schulick R, et al. Pancreatic cancer. Lancet, 2011, 378: $607-620$

3 Enzler T, Bates S. Clinical trials in pancreatic cancer: A long slog. Oncol, 2017, 22: 1424-1426

4 Ansari D, Tingstedt B, Andersson B, et al. Pancreatic cancer: Yesterday, today and tomorrow. Future Oncol, 2016, 12: 1929-1946

5 Han X, Li Y, Xu Y, et al. Reversal of pancreatic desmoplasia by reeducating stellate cells with a tumour microenvironment-activated nanosystem. Nat Commun, 2018, 9: 3390

6 Neoptolemos JP, Kleeff J, Michl P, et al. Therapeutic developments in pancreatic cancer: Current and future perspectives. Nat Rev Gastroenterol Hepatol, 2018, 15: 333-348

7 Shore S, Raraty MGT, Ghaneh P, et al. Chemotherapy for pancreatic cancer. Aliment Pharmacol Ther, 2003, 18: 1049-1069

8 Yang W, Lyu Q, Zhao J, et al. Recent advance in near-infrared/ultrasound-sensitive 2D-nanomaterials for cancer therapeutics. Sci China Mater, 2020, 63: 2397-2428

9 Justo G, Ferreira C. Coagulation and cancer therapy: The potential of natural compounds. Curr Genom, 2005, 6: 461-469

10 Tieken C, Versteeg HH. Anticoagulants versus cancer. Thrombosis Res, 2016, 140: S148-S153

11 Ansari D, Ansari D, Andersson R, Andren-Sandberg A. Pancreatic cancer and thromboembolic disease, 150 years after trousseau. Hepatobil Surg Nutr, 2015, 4: 325-335

12 Campello E, Ilich A, Simioni P, et al. The relationship between pancreatic cancer and hypercoagulability: A comprehensive review on epidemiological and biological issues. Br J Cancer, 2019, 121: 359-371

13 Khorana AA, Fine RL. Pancreatic cancer and thromboembolic disease. Lancet Oncol, 2004, 5: 655-663
14 Mast C, Ramanathan RK, Feinstein DI, et al. Disseminated intravascular coagulation secondary to advanced pancreatic cancer treated successfully with combination chemotherapy. Oncology, 2014, 87: 266-269

15 Adiseshaiah PP, Crist RM, Hook SS, et al. Nanomedicine strategies to overcome the pathophysiological barriers of pancreatic cancer. Nat Rev Clin Oncol, 2016, 13: 750-765

16 Lei Y, Tang L, Xie Y, et al. Gold nanoclusters-assisted delivery of NGF siRNA for effective treatment of pancreatic cancer. Nat Commun, 2017, 8: 15130

17 Xiong Z, Shen M, Shi X. Dendrimer-based strategies for cancer therapy: Recent advances and future perspectives. Sci China Mater, 2018, 61: 1387-1403

18 Dai T, Ye F, Hu P, et al. A strategy for enhanced tumor targeting of photodynamic therapy based on Escherichia coli-driven drug delivery system. Sci China Mater, 2021, 64: 232-240

19 Conyers J. Biomedical applications of functionalized fullerene-based nanomaterials. Int J Nanomed, 2009, 4: 261

20 Wang T, Wang C. Functional metallofullerene materials and their applications in nanomedicine, magnetics, and electronics. Small, 2019, 15: 1901522

21 Chen C, Xing G, Wang J, et al. Multihydroxylated [Gd@ $\left.\mathrm{C}_{82}(\mathrm{OH})_{22}\right]_{n}$ nanoparticles: Antineoplastic activity of high efficiency and low toxicity. Nano Lett, 2005, 5: 2050-2057

22 Li X, Zhen M, Deng R, et al. Rf-assisted gadofullerene nanoparticles induces rapid tumor vascular disruption by down-expression of tumor vascular endothelial cadherin. Biomaterials, 2018, 163: 142-153

23 Zhen M, Shu C, Li J, et al. A highly efficient and tumor vasculartargeting therapeutic technique with size-expansible gadofullerene nanocrystals. Sci China Mater, 2015, 58: 799-810

24 Meng H, Xing G, Sun B, et al. Potent angiogenesis inhibition by the particulate form of fullerene derivatives. ACS Nano, 2010, 4: 2773-2783

25 Yang D, Zhao Y, Guo H, et al. $\left[\mathrm{Gd} @ \mathrm{C}_{82}(\mathrm{OH})_{22}\right]_{n}$ nanoparticles induce dendritic cell maturation and activate Th1 immune responses. ACS Nano, 2010, 4: 1178-1186

26 Zhang $\mathrm{Y}$, Shu $\mathrm{C}$, Zhen $\mathrm{M}$, et al. A novel bone marrow targeted gadofullerene agent protect against oxidative injury in chemotherapy. Sci China Mater, 2017, 60: 866-880

27 Meng H, Xing G, Blanco E, et al. Gadolinium metallofullerenol nanoparticles inhibit cancer metastasis through matrix metalloproteinase inhibition: Imprisoning instead of poisoning cancer cells. NanoMedNanotechnol Biol Med, 2012, 8: 136-146

28 Li X, Wang C. The potential biomedical platforms based on the functionalized Gd@C $\mathrm{C}_{82}$ nanomaterials. View, 2020, 1: e7

29 Deng R, Wang Y, Zhen M, et al. Real-time monitoring of tumor vascular disruption induced by radiofrequency assisted gadofullerene. Sci China Mater, 2018, 61: 1101-1111

30 Pan Y, Wang L, Kang S, et al. Gd-metallofullerenol nanomaterial suppresses pancreatic cancer metastasis by inhibiting the interaction of histone deacetylase 1 and metastasis-associated protein 1. ACS Nano, 2015, 9: 6826-6836

31 Kang S, Zhou G, Yang P, et al. Molecular mechanism of pancreatic tumor metastasis inhibition by $\mathrm{Gd} @ \mathrm{C}_{82}(\mathrm{OH})_{22}$ and its implication for de novo design of nanomedicine. Proc Natl Acad Sci USA, 2012, 109: 15431-15436

32 Liu Y, Jiao F, Qiu Y, et al. The effect of $\mathrm{Gd} @ \mathrm{C}_{82}(\mathrm{OH})_{22}$ nanoparticles on the release of Th1/Th2 cytokines and induction of TNF- $\alpha$ mediated cellular immunity. Biomaterials, 2009, 30: 3934-3945

33 Zhou $\mathrm{Y}$, Deng $\mathrm{R}$, Zhen $\mathrm{M}$, et al. Amino acid functionalized gadofullerene nanoparticles with superior antitumor activity via destruction of tumor vasculature in vivo. Biomaterials, 2017, 133: 107-118

34 Xia S, Li J, Zu M, et al. Small size fullerenol nanoparticles inhibit thrombosis and blood coagulation through inhibiting activities of thrombin and FXa. NanoMed-Nanotechnol Biol Med, 2018, 14: 929939

35 Li X, Zhen M, Zhou C, et al. Gadofullerene nanoparticles reverse dysfunctions of pancreas and improve hepatic insulin resistance for type 2 diabetes mellitus treatment. ACS Nano, 2019, 13: 8597-8608

36 Li D, Xie K, Wolff R, et al. Pancreatic cancer. Lancet, 2004, 363: 1049_ 
1057

37 Burris 3rd HA, Moore MJ, Andersen J, et al. Improvements in survival and clinical benefit with gemcitabine as first-line therapy for patients with advanced pancreas cancer: A randomized trial. J Clin Oncol, 1997, 15: 2403-2413

38 Rossi ML, Rehman AA, Gondi CS. Therapeutic options for the management of pancreatic cancer. World J Gastroenterol, 2014, 20: 1114211159

39 Wolberg AS. Thrombin generation and fibrin clot structure. Blood Rev, 2007, 21: 131-142

40 Habib M, Saif M. Thromboembolism and anticoagulation in pancreatic cancer. J Pancreas, 2013, 14: 135-137

41 Yang Y, Stang A, Schweickert PG, et al. Thrombin signaling promotes pancreatic adenocarcinoma through PAR-1-dependent immune evasion. Cancer Res, 2019, 79: 3417-3430

42 Tsopanoglou NE, Maragoudakis ME. Role of thrombin in angiogenesis and tumor progression. Semin Thromb Hemost, 2004, 30: 63-69

43 Green D, Karpatkin S. Role of thrombin as a tumor growth factor. Cell Cycle, 2010, 9: 656-661

$44 \mathrm{Hu} \mathrm{L}$, Ibrahim S, Liu C, et al. Thrombin induces tumor cell cycle activation and spontaneous growth by down-regulation of $\mathrm{p} 27 \mathrm{Kip} 1$, in association with the up-regulation of Skp2 and MiR-222. Cancer Res, 2009, 69: 3374-3381

45 Shirai Y, Uwagawa $\mathrm{T}$, Shiba $\mathrm{H}$, et al. Recombinant thrombomodulin suppresses tumor growth of pancreatic cancer by blocking thrombininduced PAR1 and NF- $\mathrm{BB}$ activation. Surgery, 2017, 161: 1675-1682

46 Nakchbandi IA, Löhr JM. Coagulation, anticoagulation and pancreatic carcinoma. Nat Rev Gastroenterol Hepatol, 2008, 5: 445-455

47 Sudha T, Yalcin M, Lin HY, et al. Suppression of pancreatic cancer by sulfated non-anticoagulant low molecular weight heparin. Cancer Lett, 2014, 350: 25-33

Acknowledgements This work was supported by the National Major Scientific Instruments and Equipments Development Project (ZDYZ2015-2), the Key Research Program of the Chinese Academy of Sciences (QYZDJSSW-SLH025), and the National Natural Science Foundation of China (51902313).

Author contributions Li X, Zhen $\mathrm{M}$ and Wang $\mathrm{C}$ designed and performed the experiments, analyzed the data, and co-wrote the manuscript. Yu M and Zhou $\mathrm{C}$ helped with the proteomic analysis. Li L helped with AFM analysis. Shu $\mathrm{C}$ and Bai $\mathrm{C}$ advised on the experimental results. All authors discussed the results and commented on the manuscript.

Conflict of interest The authors declare that they have no conflict of interest.

Supplementary information Other experimental details and supporting data are available in the online version of the paper.

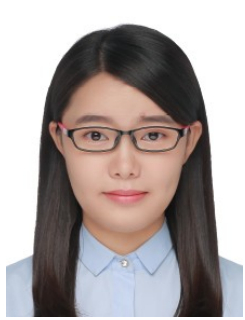

Xue Li received her $\mathrm{PhD}$ degree from the Institute of Chemistry, Chinese Academy of Sciences (CAS) in 2018. She is now an assistant professor at the Institute of Chemistry, CAS. Her research interests include the pharmacokinetics study of fullerenes, and the biomedical applications of fullerenes.

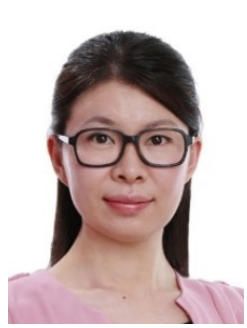

Mingming Zhen obtained her PhD degree in physical chemistry from the Institute of Chemistry, CAS in 2014. She is now an associate professor at the Institute of Chemistry, CAS. Her research interests include biomedical applications of fullerene and gadofullerenes, especially in therapeutics for tumor, metabolic disorders, and neurodegenerative diseases.

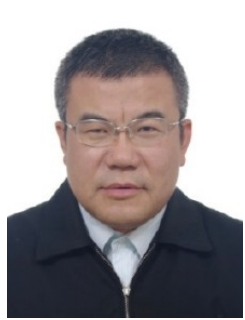

Chunru Wang received his $\mathrm{PhD}$ degree from Dalian Institute of Chemistry Physics, CAS in 1992. He is a professor at the Institute of Chemistry, CAS. His research interests include industrial preparation of fullerenes and endohedral fullerenes, structure and physicochemical properties of fullerene and metallofullerene materials, biomedical applications of fullerene nanoparticles. Currently, he focuses on the transformation of fullerene research achievements and fullerene drug development.

\section{钝金属富勒烯纳米材料在原位胰腺癌治疗中延长生 存率并降低凝血酶表达}

李雪 ${ }^{1,2}$, 甄明明 $1,2^{*}$, 俞梅兰 ${ }^{3}$, 周辰 ${ }^{1,2}$, 李蕾 ${ }^{1,2}$, 舒春英 ${ }^{1,2}$, 王春儒 ${ }^{1,2^{*}}$, 白春礼 1,2

摘要 胰腺癌是一种恶性程度很高的癌症，其5年生存率低于 $8 \%$. 由于 胰腺癌具有高度纤维化、过度结缔组织增生的微环境，限制了抗肿瘤 药物的渗透, 并且大多数现有抗胰腺癌药物具有严重的副作用, 因此, 仍急需研发更加安全高效地治疗胰腺癌的药物来延长患者的生存时 间，改善预后. 本文基于钝金属纳米颗粒(GFNPs)发展了一种高效安全 的治疗原位肤腺癌的策略.GFNPs不仅能够显著抑制原位胰腺癌的生 长, 存在浓度依赖性, 而且能够显著延长患癌小鼠的生存期. 此外, 本 文利用蛋白质组学的手段, 对GFNPs治疗原位胰腺癌过程中的蛋白变 化进行了系统分析, 发现GFNPs能够改善凝血信号通路异常, 下调胰腺 癌组织凝血酶的表达. GFNPs不仅能够抑制凝血酶基因及蛋白水平的 表达，还能够抑制凝血酶的活性. 更重要的是，GFNPs几乎无明显的毒 副作用, 优于常用治疗胰腺癌药物吉西他滨. 因此, GFNPs不仅能够高 效安全地治疗胰腺癌, 还有望改善癌症相关血栓栓塞类疾病的发展. 